\title{
Principios quirúrgicos en fracturas de platillos tibiales con compromiso de columna posterior
}

\section{Surgical Principles in Posterior Column Tibial Plateau Fractures}

\author{
Pedro Díaz Allende ${ }^{1}$ Maximiliano Scheu Gonçalves ${ }^{2,3}$ Xabier Carredano González ${ }^{3}$ \\ Oscar Colmenares Sandoval ${ }^{1}$ Claudio Yáñez Lagos ${ }^{4}$ Rodrigo Donoso Martínez ${ }^{5}$ \\ Luis O’Connell Godoy ${ }^{6}$ David Figueroa Poblete ${ }^{2}$
}

${ }^{1}$ Fellow Cirugía Artroscópica y Reconstructiva de Rodilla, Clínica Alemana de Santiago, Universidad del Desarrollo / Hospital Clínico Mutual de Seguridad de Santiago, Chile

2 Unidad de Rodilla, Clínica Alemana de Santiago, Chile

${ }^{3}$ Unidad de Rodilla, Hospital Clínico Mutual de Seguridad de Santiago, Chile

${ }^{4}$ Traumatólogo Estadía en Capacitación en el Departamento de Ortopedia y Traumatología, Clínica Alemana de Santiago, Chile

${ }^{5}$ Becado de Ortopedia y Traumatología, Clínica Alemana de Santiago Universidad del Desarrollo, Chile

${ }^{6}$ Médico-Cirujano. Estadía en Capacitación en el Departamento de Ortopedia y Traumatología, Hospital Clínico Mutual de Seguridad de Santiago, Chile

Address for correspondence Pedro Díaz Allende, MD, Departamento Ortopedia y Traumatología, Clínica Alemana de Santiago. Av. Vitacura 5951, Vitacura, Región Metropolitana, Santiago, Chile (e-mail: drpedrodiaz@gmail.com).

Rev Chil Ortop Traumatol 2018;59:22-34.

\section{Resumen \\ Palabras Clave \\ - abordaje posterior directo \\ - abordaje quirúrgico \\ - columna posterior \\ - columna posterolateral \\ - columna posteromedial \\ - fractura de platillos tibiales}

Las fracturas de platillos tibiales son lesiones complejas que incluyen una variedad de patrones morfológicos cada vez mejor caracterizados en la literatura. Históricamente, los esquemas de clasificación se han basado en evaluar los rasgos de fractura en el plano frontal y las técnicas quirúrgicas en lograr la fijación de esos fragmentos, sin tomar en consideración el compromiso óseo que ocurre en la región posterior de los platillos tibiales. Con el advenimiento de la clasificación columnar basada en tomografía computada, se han logrado desarrollar estrategias de fijación optimizada, dando cada vez más relevancia a la columna posterior. Este artículo realiza una revisión extensa de la literatura, otorgando los fundamentos quirúrgicos que explican la importancia del tratamiento específico de la columna posterolateral y posteromedial, con el fin de restablecer la biomecánica normal de la rodilla y el razonamiento quirúrgico de las diversas vías de abordaje específicas para una reducción y osteosíntesis satisfactoria de esos fragmentos. received

December 17, 2017

accepted

March 12, 2018
DOI https://doi.org/

10.1055/s-0038-1641563. ISSN 0716-4548.
Copyright @ 2018 by Thieme Revinter

Publicações Ltda, Rio de Janeiro, Brazil
License terms

(c) $(1) \$$ 


\begin{abstract}
Keywords

- direct posterior approach

- posterior column

- posterolateral column

- posteromedial column

- surgical approach

- tibial plateau fracture

Tibial plateau fractures are complex injuries which include a variety of morphological patterns that have been increasingly better characterized in the literature. Historically, classifications have focused on description of fracture patterns in the frontal plane, while surgical techniques have focused on reduction of these fragments not considering the osseous defects that occur on the posterior region of the tibial plateau. With new CT scan column based classifications, strategies to optimize fixation have been developed, giving relevance to the posterior column. This article is an exhaustive review of the literature, providing the surgical foundations that explain the importance of specific treatment of the posterolateral and posteromedial column, aiming to restore normal knee biomechanics. Furthermore, this article provides the diverse specific surgical approaches rationale for a satisfactory open reduction and internal fixation of these fragments.
\end{abstract}

\section{Evolución de los Sistemas de Clasificación, Incidencia y Morfología de los Fragmentos Posteriores}

El manejo de las fracturas de platillos tibiales ha evolucionado en la medida que lo han hecho sus resultados, lo que ha influido en la evolución de los sistemas de clasificación, a fin de lograr describir de la mejor forma posible la localización y características de los fragmentos, permitiendo una planificación preoperatoria adecuada y orientando el tratamiento.

Históricamente, las fracturas que afectan la región posterior de los platillos tibiales han sido subestimadas y resueltas de forma inadecuada.

La clasificación de Schatzker, ${ }^{1}$ publicada en 1979, divide las fracturas en 6 tipos y es la más utilizada a nivel internacional. En 1987, el grupo AO presentó su clasificación alfanumérica, ${ }^{2}$ la cual aporta mayor detalle respecto a las características del compromiso óseo, sin embargo, posee una importante variabilidad interobservador. ${ }^{3}$ Ambas clasificaciones no permiten una valoración de la integridad de la superficie articular posterior debido a que están basadas en una evaluación radiográfica en el plano frontal.

La tomografía computada (TC) y las reconstrucciones tridimensionales han ampliado el entendimiento de esas lesiones al otorgar una perspectiva en los planos coronal, sagital y axial, agregando información invaluable para el tratamiento, favoreciendo el desarrollo de nuevas clasificaciones y mejorando la confiabilidad intra e interobservador, respecto de las características morfológicas de esos fragmentos. ${ }^{4,5}$

En 2010, Luo y cols ${ }^{6}$ describen el concepto tricolumnar de los platillos tibiales (lateral, medial y posterior), permitiendo guiar de manera más reproducible la estrategia quirúrgica. Eso renovó el interés en abordajes específicos para el tratamiento de fracturas en la región posterior de la rodilla descritos previamente por pioneros como Gossling y Peterson, ${ }^{7}$ Georgeadis, $^{8}$ De Boeck y Opdecam, ${ }^{9}$ Lobenhoffer, ${ }^{10}$ Carlson, ${ }^{11}$ y Galla, ${ }^{12}$ dando paso al desarrollo de nuevas vías de acceso a la región posterior de la rodilla.
Bajo este sistema de clasificación, Yang y $\operatorname{cols}^{13}$ en su análisis tomográfico de 525 fracturas, encontró un 28,8\% de compromiso de la columna posterior. De ellas, el $72,8 \%$ se encuentran asociadas a la lesión de otra columna. Los 3 tipos de fractura con mayor asociación a compromiso de columna posterior fueron las lesiones Schatzker VI $(76,1 \%), \mathrm{V}(51,2 \%)$ y IV $(22,4 \%)$. Otras series con menor número de casos, ${ }^{14,15}$ caracterizan el fragmento posteromedial aislado (en fracturas Schatzker IV) con incidencias entre 42,7\% y 47,8\%.

Las fracturas de platillo tibial Schatzker II son las de mayor frecuencia clínica. Zhai y cols ${ }^{16}$ evaluaron sus características morfológicas en un estudio basado en TC donde reclutaron 140 pacientes, encontrando que la incidencia de fragmentos posterolaterales desplazados era de un 10,7\%.

En 2014, Chang y cols ${ }^{17}$ dividen las fracturas de platillos tibiales en 4 columnas (anteromedial, anterolateral, posteromedial y posterolateral), con el fin de mejorar la caracterización de los fragmentos posteriores (-Fig. 1). Además, se ha demostrado que esa clasificación tiene los más altos porcentajes de acuerdo intra e interobservador, con diferencias estadísticamente significativas respecto a las clasificaciones clásicas. ${ }^{18}$ El estudio de Yang y cols ${ }^{13}$ permitió conocer que del total de lesiones de la columna posterior, el $45,7 \%$ compromete la columna posteromedial, el 35,1\% la columna posterolateral y un $19,2 \%$ ambas columnas posteriores.

Barei y col ${ }^{19}$ y luego Higgins y col$^{20}$ caracterizaron de manera completa el fragmento posteromedial mediante TC (-Tabla 1). Tiene la forma de un cono invertido. La superficie promedio comprometida representa más de la mitad de la superficie articular del platillo medial (58\%) y entre el $23 \%$ y el 25\% de la superficie articular total de los platillos tibiales, con una altura promedio del fragmento en el plano sagital de $42 \mathrm{~mm}$ a $45 \mathrm{~mm}$. La angulación axial de la línea de fractura es en promedio de $9^{\circ}$ a $21^{\circ}$, con el fragmento generalmente en rotación externa. Eso las convierte en fracturas cizallantes en el plano coronal altamente inestables. La mayoría (55\%) presenta desplazamientos inaceptables para el manejo conservador (>5 mm). El $74 \%$ de las fracturas con compromiso de ambos platillos tibiales presentan un fragmento posteromedial. ${ }^{19}$ 


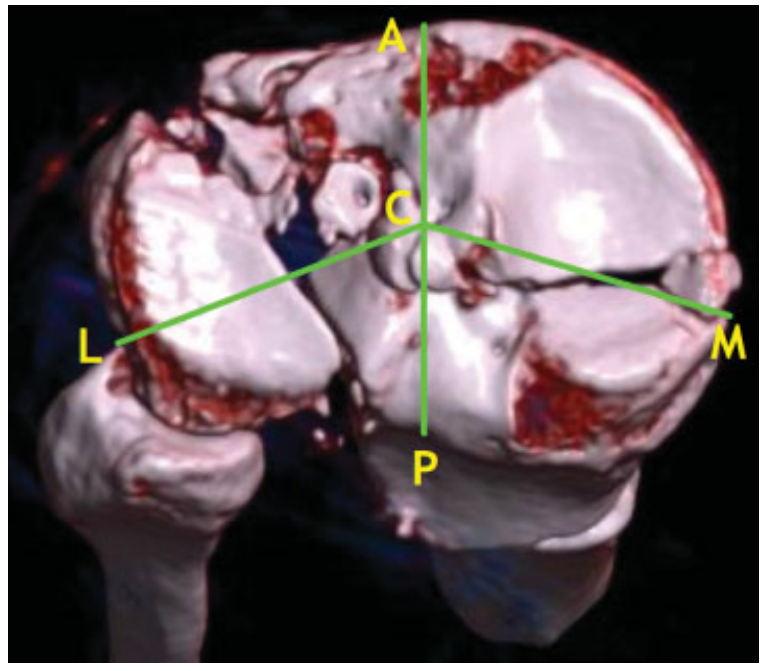

Fig. 1 Concepto Columnar en la Clasificación de Fracturas de Platillos Tibiales: Las columnas están separadas por líneas conectoras llamadas CA, CL, CM y CP. El punto $C$ representa el centro de la rodilla (punto medio de ambas espinas tibiales). A, representa la tuberosidad anterior de la tibia. $\mathrm{M}$, representa el extremo posteromedial de la tibia proximal. L, representa la proyección tibial del punto más anterior de la cabeza fibular. P, es el surco posterior que divide ambos platillos tibiales y bisecta la columna posterior en columnas posteromedial (PM) y posterolateral (PL).

Más recientemente, Zhu y $\operatorname{cols}^{21}$ y luego Sohn y $\operatorname{cols}^{22}$ lograron similar caracterización del fragmento posterolateral mediante TC (Tabla 1). También presenta forma de cono invertido. La superficie promedio comprometida representa entre el $14,5 \%$ al $16,7 \%$ de la superficie articular total de los platillos tibiales y aproximadamente un tercio del platillo tibial lateral. La altura promedio del fragmento es de $29,6 \mathrm{~mm}$ a $31 \mathrm{~mm}$ en el plano sagital. La angulación axial de la línea de fractura es en promedio de $13^{\circ}$ y en general en rotación externa, lo que las convierte en fracturas cizallantes e inestables en el plano coronal. El 40,4\% tiene desplazamientos mayores a $5 \mathrm{~mm}$.

En 2016, Krause y cols, ${ }^{23,24}$ caracterizan el compromiso articular en 8 y luego en 10 columnas, en un intento de mejorar la estrategia quirúrgica de esas lesiones. Su utilidad clínica respecto a las clasificaciones ya descritas debe ser analizada con el tiempo y estudios clínicos prospectivos. ${ }^{25,26}$

La principal limitación de los sistemas de clasificación columnar es que solamente proveen información respecto al compromiso de la superficie articular tibial proximal. Sin embargo, existen otros aspectos importantes que deben ser considerados con el fin de realizar una evaluación completa de la lesión, como el compromiso metafisio-diafisiario y el estado de las partes blandas. Durante la fijación de ese tipo de fracturas, es importante identificar su ápice, lo cual guiará el abordaje requerido y la posición de la placa con el fin de prevenir el deslizamiento del fragmento. Esos aspectos pueden ser evaluados adicionando una clasificación clásica como la de Schatzker ${ }^{1}$ y una Clasificación del estado de partes blandas como la de Tscherne para fracturas cerradas $^{27}$ o Gustilo-Anderson para fracturas expuestas. ${ }^{28}$

\section{Mecanismos de Lesión y Biomecánica de la Columna Posterior de los Platillos Tibiales}

Los patrones de fractura del platillo tibial dependen fundamentalmente de 2 factores: la posición de la rodilla al momento de la lesión y la dirección de la fuerza deformante. La clasificación en columnas permite la evaluación del mecanismo de lesión. La fractura de la columna posterior es producida por una carga axial proveniente de los cóndilos femorales en la porción posterior de los platillos tibiales cuando la rodilla se encuentra en algún grado de flexión y rotación femoral, determinando el hundimiento o cizallamiento del fragmento. Las fuerzas deformantes determinan el compromiso columnar posterolateral (valgo) o posteromedial (varo).

Estudios biomecánicos ${ }^{29-31}$ han demostrado que el contacto tibio femoral se desplaza hacia posterior en la medida que aumenta la flexión de rodilla (rollback femoral). En grados iniciales $\left(30^{\circ}\right)$ e intermedios de flexión $\left(60^{\circ}\right)$, un trauma con suficiente carga axial produce generalmente una fractura cizallante en el plano coronal. ${ }^{21,32}$ En grados más profundos de flexión, se ha demostrado en modelos

Tabla 1 Comparación de las principales características morfológicas de los fragmentos posteromedial y posterolateral en fracturas de platillos tibiales

\begin{tabular}{|l|l|l|}
\hline Característica & Fragmento posteromedial & Fragmento posterolateral \\
\hline Mecanismo de lesión & $\begin{array}{l}\text { Carga axial, varo y grados variables de } \\
\text { flexión y rotación femoral interna }\end{array}$ & $\begin{array}{l}\text { Carga axial, valgo y grados variables } \\
\text { de flexión y rotación femoral externa }\end{array}$ \\
\hline Forma & Cono invertido & \multicolumn{2}{|l|}{$\begin{array}{l}14,5 \%-16,7 \% \text { del área articular total } \\
\text { Superficie articular comprometida }\end{array}$} & $\begin{array}{l}23 \%-25 \% \text { del área articular total } \\
58 \% \text { del platillo tibial medial }\end{array}$ & $29,6-31 \mathrm{~mm}$ \\
\hline Altura del fragmento en el plano sagital & $42-45 \mathrm{~mm}$ & $13^{\circ}$ (rotación externa) \\
\hline Angulación axial de la línea de fractura & $9^{\circ}-21^{\circ}$ (rotación externa) \\
\hline Patrón de fractura predominante & Coronal cizallante & $40,4 \%$ \\
\hline $\begin{array}{l}\text { Incidencia de desplazamiento } \\
\text { inaceptable }(>5 \mathrm{~mm})\end{array}$ & $55 \%$ & \\
\hline
\end{tabular}


cadavéricos $^{21}$ que el patrón más frecuente de fractura es el hundimiento asociado a conminución de la columna posterior.

La inestabilidad articular es directamente proporcional al grado de flexión de la rodilla y la orientación de la línea de fractura en el plano axial. ${ }^{32}$ Eso se traduce en subluxación posterior del fémur durante la marcha $\mathrm{y}$ artrosis postraumática de no mediar una reducción anatómica y osteosíntesis específica del fragmento posterior. Cuellar y $\mathrm{col}^{33}$ en el año 2015, confirman el comportamiento inestable del fragmento posteromedial en distintas condiciones de carga y rango articular, independiente del tamaño que él tenga o su grado de desplazamiento.

El ángulo del fragmento articular mayor (AFAM) $)^{19,20,22}$ es aquel que se forma entre una línea tangencial que mejor se ajuste al rasgo mayor de fractura que compromete la columna posterior en el plano axial y el eje femoral condilar posterior (EFCP), ${ }^{19,20,22}$ el cual representa una tangente a los cóndilos femorales posteriores en el plano axial a nivel de los epicóndilos (-Fig. 2). Ese eje es neutral e independiente del eje horizontal de la imagen y permite evitar errores de medición por eventual rotación interna o externa de la extremidad dentro del escáner. El AFAM es positivo si el fragmento está rotado interno en relación al EFCP y negativo si está rotado externo en relación a ese eje. Su
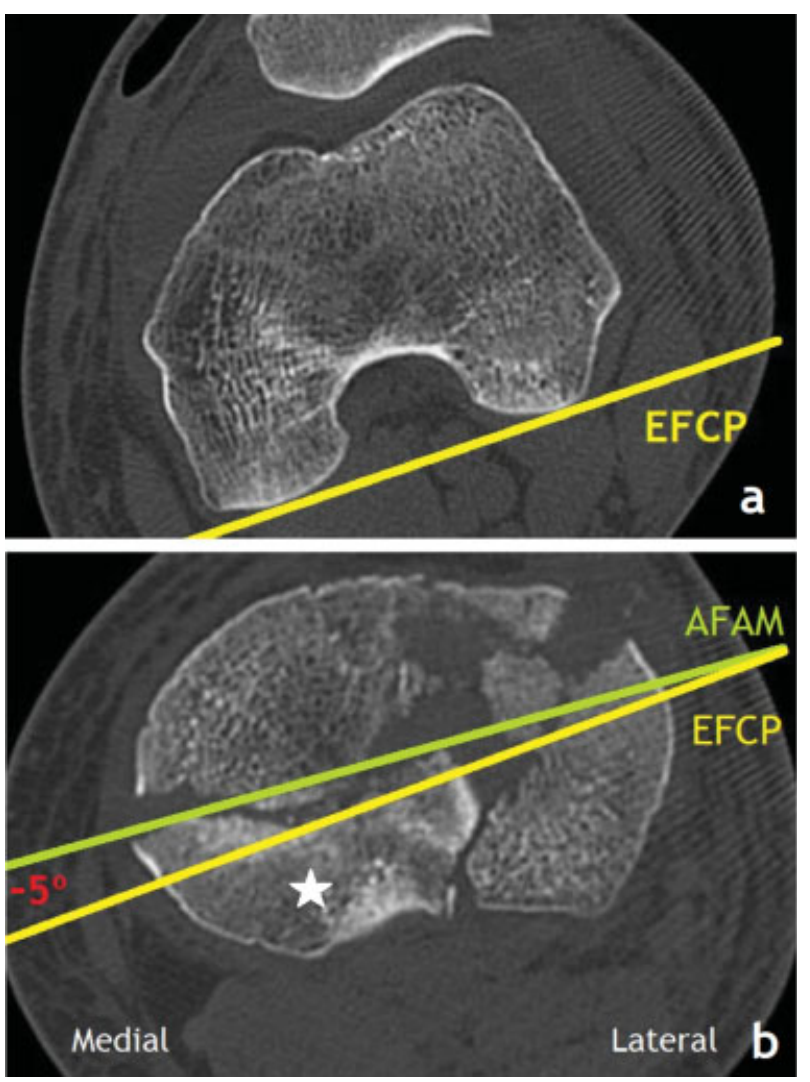

Fig. 2 Medición angular en la caracterización morfológica de los fragmentos de la columna posterior: a. El eje femoral condilar posterior (EFCP), se utiliza como línea de referencia para determinar el ángulo de rotación de la extremidad inferior relativa al plano horizontal. b. El ángulo del fragmento articular mayor (AFAM) se determina midiendo el ángulo entre el EFCP y la línea de fractura principal. El fragmento posteromedial (estrella blanca) se encuentra rotado externo en $5^{\circ}$, calificando como fractura coronal. importancia radica en que define el concepto de fractura coronal en el fragmento posterior si su valor se encuentra entre $-45^{\circ} \mathrm{y}+30^{\circ}$, lo que tiene repercusiones en la estabilidad de la lesion. ${ }^{34}$ Además, describe cuán posterior se encuentra localizado el fragmento, orienta respecto al abordaje necesario para su reducción y la dirección en la que debiesen ingresar los tornillos para su óptima fijación (perpendiculares al AFAM).

\section{Principios del Tratamiento Quirúrgico}

Los principios del manejo de toda fractura de platillos tibiales se basa en la reconstrucción anatómica de la superficie articular, restableciendo el eje mecánico y longitud de la tibia en el plano coronal y sagital, asociado a estabilidad absoluta para permitir movilización precoz y evitar el colapso articular. Para poder lograr esos objetivos, es necesario tomar en consideración una evaluación columnar de la articulación y la extensión distal de los rasgos de fractura. El grupo de Luo ${ }^{6,35}$ define como reducción inadecuada: escalones articulares $>2 \mathrm{~mm}$; ángulo tibial medial proximal (ATMP) $\geq 95^{\circ} \mathrm{o} \leq 80^{\circ} \mathrm{y}$ slope tibial $\geq 15^{\circ} \mathrm{o} \leq-5^{\circ}$. A esos criterios debemos agregar por su importancia, un ensanchamiento del platillo tibial $>5 \mathrm{~mm} .{ }^{36}$ El ATMP está formado por la intersección del eje mecánico tibial con una línea tangencial que conecta los extremos lateral y medial de los platillos tibiales en una radiografía AP de tibia. ${ }^{37}$ El slope tibial está formado por la intersección de una perpendicular al eje mecánico de la tibia con la línea que conecta el borde anterior y posterior del platillo tibial medial en una radiografía lateral de tibia. ${ }^{38}$

Existe controversia respecto a la relación entre el grado de escalón articular y riesgo de artrosis postraumática en fracturas de platillos tibiales. Giannoudis y $\operatorname{cols}^{39}$ realizaron una revisión sistemática al respecto, encontrando que la mayoría de los autores están de acuerdo que el rango de escalón aceptable se encuentra entre 2 y $10 \mathrm{~mm}$.

El reconocimiento del mecanismo de lesión permite dividir las fracturas columnares en lesiones por compresión y por falla de tensión. La placa primaria de sostén debe aplicarse para revertir las fuerzas deformantes compresivas en las columnas afectadas por ese mecanismo. Una placa de soporte secundaria está indicada para estabilizar columnas que han fallado debido a fuerzas de tensión si son conminutas o inestables. ${ }^{35}$ Debido a lo pequeño de los fragmentos posteriores, se recomienda la utilización de sistemas de fijación 3,5/4,5 mm en lugar de los convencionales 4,5/6,5 mm. ${ }^{6}$

La definición de perdida de reducción no es clara para ese tipo de fracturas. Luo y col$^{35}$ la define como un cambio en el alineamiento tibial $>5^{\circ}$ en varo o valgo y/o depresión articular $\geq 2 \mathrm{~mm}$ cuando se compara la primera radiografía postoperatoria con aquella tomada al final del seguimiento. Kim y col$^{34}$ agrega ensanchamiento del platillo tibial $>5 \mathrm{~mm}$, difiriendo con Luo en la depresión de la superficie articular (> $3 \mathrm{~mm}$ ). Morfológicamente, según diversos estudios, las fracturas de columna posterior son predominantemente coronales. $^{19-22}$ La conminución es un indicador de mal pronóstico ampliamente aceptado. Kim y $\operatorname{cols}^{34}$ confirman 
que esos 2 factores son predictores estadísticamente significativos de perdida de reducción en cirugía de platillos tibiales, siendo relevante la fijación específica de ese tipo de fragmentos. En su estudio, que no incluyó fijación específica de la columna posterior, observó en promedio $29 \%$ de pérdidas de reducción en fracturas $\mathrm{AO}$ : $\mathrm{B}$ y $\mathrm{C}$, incluso con uso de doble placa en los casos con compromiso de ambos platillos.

La columna posterior del platillo tibial presenta una anatomía irregular y compleja. El grupo de Luo ${ }^{40}$ caracterizó su morfología normal. En el plano axial, presenta una forma de doble arco asimétrico (posteromedial y posterolateral). En el plano sagital, 2 angulaciones significativas consecutivas en el margen posterior de la tibia proximal y en el plano coronal, una depresión elíptica bajo la inserción del ligamento cruzado posterior (entre ambos arcos) conocida como champagne glass drop off. 41

La mayoría de las placas de osteosíntesis no presentan una adaptación específica para esa columna, por lo que la elección del sistema de fijación interna varía respecto a la experiencia del cirujano. Se han descrito en la literatura diversas opciones: placas en $\mathrm{T}, \mathrm{L}$ o rectas (bloqueadas o convencionales), placas tercio de tubo, placas de reconstrucción y recientemente la producción de placas específicas para la columna posterior. ${ }^{42}$

Los principios del manejo postoperatorio se rigen por las mismas reglas comunes a todas las fracturas de platillos tibiales. Idealmente, lograr rangos de movilidad completo durante las primeras 4 semanas, con restricción de carga de peso en promedio durante 12 semanas, dependiendo de la consolidación (definida radiológicamente como la unión de al menos 3 corticales durante el periodo de seguimiento), y características específicas de cada paciente.

\section{Racionalidad de los Abordajes Posteriores Directos}

Los abordajes quirúrgicos tradicionales no proveen suficiente exposición para lesiones de la columna posterior. El intento de realizar ampliación de esos abordajes (diseñados para lesiones de columnas anterolateral y anteromedial), se puede asociar a la lesión injustificada de partes blandas, extensión del tiempo quirúrgico y complicaciones postoperatorias derivadas de una fijación insuficiente y dificultosa. Los métodos de reducción indirectos están comúnmente asociados a la incongruencia articular.

La cirugía en la región posterior de la rodilla puede ser desafiante debido a la presencia de estructuras neurovasculares en riesgo, incluyendo el nervio tibial, la arteria y vena poplítea, el nervio cutáneo sural lateral y medial, el paquete vascular tibial anterior (conocido como trifurcación) y el nervio peroneo común, por lo que es poco utilizado en la práctica habitual. Un conocimiento anatómico detallado de la localización de esas estructuras y sus posibles variantes, es fundamental para un abordaje posterior seguro y exitoso. ${ }^{43,44}$

Múltiples estudios han confirmado la importancia de los abordajes posteriores y su correlación con mejores resultados clínicos. ${ }^{35,45,46}$ En esa situación, una placa de sostén posterior es el constructo biomecánicamente más estable. ${ }^{6,47,48}$ El tipo de abordaje posterior óptimo ha sido un tópico de gran interés en los últimos años (-Tabla 2).

El fragmento posteromedial a menudo se encuentra asociado a fracturas de ambos platillos tibiales. ${ }^{19,20,49,50}$ Algunos autores han sugerido que la estabilidad dada por las placas bloqueadas laterales es suficiente para estabilizar ese fragmento. ${ }^{51,52}$ Sin embargo, el fragmento posteromedial es la zona que se encuentra más alejada de la contención que otorga la placa lateral a fuerzas en el plano axial y los tornillos pueden fallar en capturar ese fragmento.

Otros autores ${ }^{53,54}$ han demostrado perdidas sustanciales de reducción con una placa bloqueada lateral aislada, dada la desventaja mecánica que implica la distancia entre el fragmento y el constructo. Estudios biomecánicos contemporáneos confirman que la asociación de una placa de sostén posteromedial $3.5 \mathrm{~mm}$ (bloqueada o convencional) provee significativamente mayor estabilidad, resistencia al desplazamiento y carga máxima para el fallo que una placa lateral aislada. ${ }^{47,55,56} \mathrm{El}$ fragmento coronal cizallante

Tabla 2 Características de los abordajes posteriores directos para el manejo de fracturas de platillos tibiales con compromiso de la columna posterior

\begin{tabular}{|l|l|l|}
\hline & Ventajas & Desventajas \\
\hline $\begin{array}{l}\text { Abordaje } \\
\text { en prono }\end{array}$ & $\begin{array}{l}\text { Reducción bajo visión directa facilitada por la (hiper) } \\
\text { extensión de rodilla. } \\
\text { Facilidad para aplicar placas de sostén y tornillos de } \\
\text { posterior a anterior. } \\
\text { Evita lesión de tendones de la pata de ganso. } \\
\begin{array}{l}\text { Puede exponer a la vez lesiones de columna } \\
\text { posteromedial y posterolateral. } \\
\text { Ideal ante compromiso aislado de la columna } \\
\text { Abosterior. }\end{array}\end{array}$ & $\begin{array}{l}\text { Riesgoso en pacientes politraumatizados, trauma } \\
\text { enceano, lesiones agudas torácicas o } \\
\text { abdominales y patología pulmonar crónica. } \\
\text { Requiere más tiempo el posicionamiento adecuado } \\
\text { del paciente. } \\
\text { Más propenso a lesionar estructuras vasculares. }\end{array}$ \\
& $\begin{array}{l}\text { Conveniente para la mayoría de las fracturas de } \\
\text { platillos tibiales. } \\
\text { Seguro en pacientes que requieren monitorización } \\
\text { anestésica estrecha. } \\
\text { Posicionamiento del paciente más sencillo. } \\
\text { Ideal ante compromiso dual de columna anterolateral } \\
\text { y posteromedial. }\end{array}$ & $\begin{array}{l}\text { Difícil insertar tornillos de posterior a anterior. } \\
\text { Pueden ocurrir problemas en la reducción de los } \\
\text { fragmentos posteriores y dificultad para mantener su } \\
\text { Requiere osteotomia del peroné para exponer el } \\
\text { fragmento posterolateral. }\end{array}$ \\
\hline
\end{tabular}


posteromedial debe reducirse anatómicamente mediante reposición exacta de su espícula cortical inferior bajo visualización directa (según lo planificado preoperatoriamente). La fijación se realiza mediante los principios generales de $\mathrm{AO}$ para una placa de sostén antideslizante, usualmente con 4 tornillos corticales de $3,5 \mathrm{~mm}, 2$ a cada lado de la espícula ósea. Se pueden instalar tornillos con técnica de compresión interfragmentaria en los orificios proximales de la placa para incrementar la compresión a lo largo de la línea de fractura (-Fig. 3).

Por otra parte, los fragmentos posterolaterales desplazados son incapaces de ser contenidos mediante placas instaladas por medio de un abordaje anterolateral tradicional, debido a la obstrucción que genera la cabeza de la fíbula y las estructuras ligamentarias que allí se insertan. Solomon y $\operatorname{cols}^{57}$ realizaron un estudio comparativo confirmando que un abordaje posterolateral directo mejora la reducción, estabilización y resultados funcionales frente a un abordaje indirecto anterolateral. Sohn y $\operatorname{cols}^{22}$ midieron la distancia promedio existente entre la cortical anterior de la cabeza de la fíbula y el punto de salida del rasgo posterolateral en la cortical lateral del platillo tibial, encontrando un valor de 10,2 $\mathrm{mm}$. Considerando los diseños de placas anatómicas proximales laterales de tibia, no parece probable poder instalar más de 1 tornillo hacia ese fragmento en la mayoría de los casos. Sassoon y cols $^{58}$ evaluaron el área de platillo tibial lateral que no logra ser contenido tras la instalación de 6 modelos de placa bloqueada lateral de tibia proximal más utilizadas en el mercado, encontrando que la distancia entre el tornillo subcondral más posterior de cada una de ellas al borde posterolateral del platillo tibial resultaba en un área no soportada por tornillos correspondiente en promedio al $40 \%$ (rango $25 \%-58 \%$ ) del platillo tibial lateral. Zhang y cols ${ }^{48}$ enfatizan que la placa de sostén posterolateral es la fijación biomecánica más resistente para contener ese tipo de lesiones. En la porción posterior de la tibia, el fragmento posterolateral presenta dimensiones promedio de $22,9 \mathrm{~mm}$ de ancho y 31,1 $\mathrm{mm}$ de largo, ${ }^{22}$ lo cual es un área suficiente para la instalación de una placa de sostén de $3,5 \mathrm{~mm}$ en esa zona que evite la subsidencia del fragmento. Con el fin de evitar lesión del paquete vascular tibial anterior con la placa a nivel posterolateral, ésa puede instalarse en posición oblicua (con su extremo distal en dirección posteromedial). Ese constructo ha demostrado ser la fijación biomecánicamente más estable para ese fragmento específico, con desplazamientos verticales bajo carga axial $\leq 1 \mathrm{~mm}^{48}$

En una revisión reciente respecto a resultados postoperatorios con el uso de abordajes directos para el
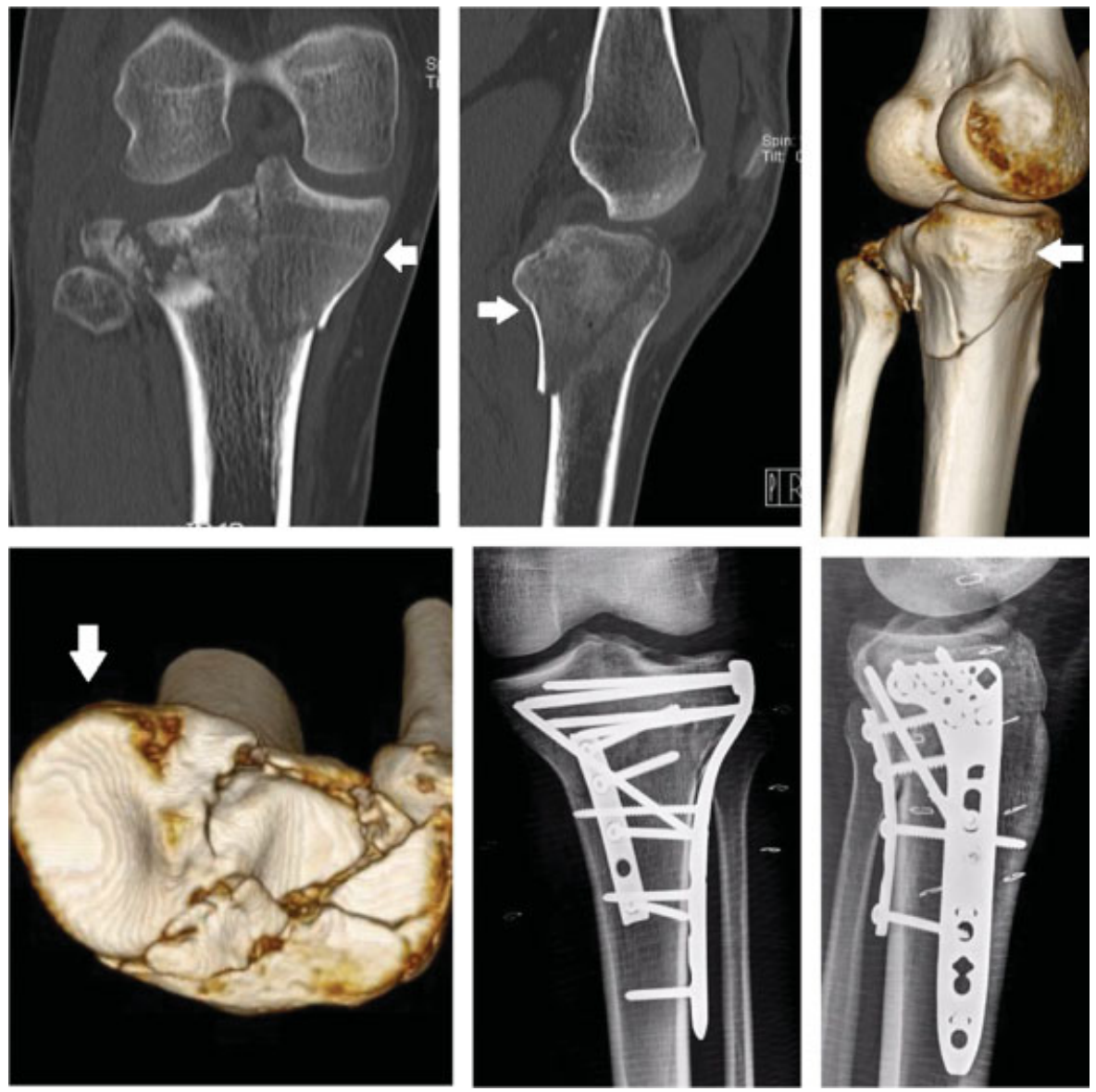

Fig. 3 Técnica de fijación de fragmentos posteriores. Fractura de platillos tibiales Schatzker $V$ con un fragmento inestable posteromedial (flecha blanca). Se realiza abordaje dual de las lesiones, iniciando la intervención con reducción del fragmento posteromedial con placa de sostén antideslizante, para luego finalizar con el manejo de la columna anterolateral y posterolateral con una placa bloqueada anatómica lateral de ángulo variable. 
manejo de fracturas que afectan la columna posterior, no hubo evidencia de mayores tasas de complicaciones respecto a los abordajes tradicionales, pero sí de mejores resultados clínicos y radiológicos. ${ }^{45}$

\section{Posicionamiento del Paciente y Secuencia de Fijación en Fracturas con Compromiso de Ambos Platillos Tibiales}

Los abordajes duales, que combinan una exposición anterolateral y posteromedial son el pilar fundamental en la mayoría de las fracturas que comprometen ambos platillos tibiales.

Generalmente, el compromiso posteromedial es tratado primero con el fin de facilitar la reconstrucción del largo y eje de la extremidad y mantener una reducción concéntrica de la articulación. Eso se debe, principalmente, a que el compartimiento femorotibial medial es el que otorga la estabilización mecánica primaria a la rodilla por sus superficies articulares altamente congruentes, ${ }^{30}$ lo que facilita la corrección de los ángulos anatómicos especialmente en el plano frontal. ${ }^{49}$ Además, en presencia de una fractura con extenso compromiso de la columna posterior, el fémur distal mantendrá su relación con las estructuras capsulares de ese fragmento, generándose una traslación posterior del fémur en el plano sagittal. ${ }^{59}$

Inicialmente, Barei y cols ${ }^{60}$ y luego Weil y cols, ${ }^{61}$ describen el abordaje posteromedial clásico en posición supina, en el contexto de fracturas con compromiso de ambos platillos tibiales, posicionando la extremidad en leve flexión de rodilla $\left(30^{\circ}\right)$, abducción y rotación externa de la cadera para permitir una exposición suficiente del fragmento posteromedial. Esos autores logran cambiar el paradigma del abordaje único mediante incisión anterior en la línea media para el manejo de ese tipo de fracturas, logrando demostrar una reducción significativa en la tasa de complicaciones e infecciones profundas mediante el abordaje dual: anterolateral y posteromedial.

Tras el advenimiento de la clasificación columnar y el tratamiento del fragmento específico, Luo y cols ${ }^{6}$ describieron la posición flotante, que consiste en posicionar al paciente en decúbito semilateral, donde la extremidad inferior afectada puede rotarse a una posición en prono cuando se realiza el abordaje posterior de rodilla y volver a una posición lateral cuando se realiza el abordaje anterolateral clásico (-Fig. 4). Esa posición ha sido utilizada por varios autores, demostrando su utilidad en fracturas complejas con compromiso de ambos platillos tibiales. ${ }^{17,62,63}$ El decúbito prono facilita la reducción del componente posteromedial. La extremidad inferior en supino tiende a asumir la posición de flexión y varo de rodilla, mientras que la reducción de ese fragmento, se facilita en extensión y valgo, lo cual es más ventajoso en prono, ya que la gravedad ayuda en la reducción indirecta y es una posición más ergonómica para el cirujano. En decúbito supino, la inserción de los implantes y la trayectoria deseada para los tornillos puede entrar en conflicto con la mesa quirúrgica, la extremidad inferior contralateral y los componentes auxiliares de posicionamiento de la extremidad.
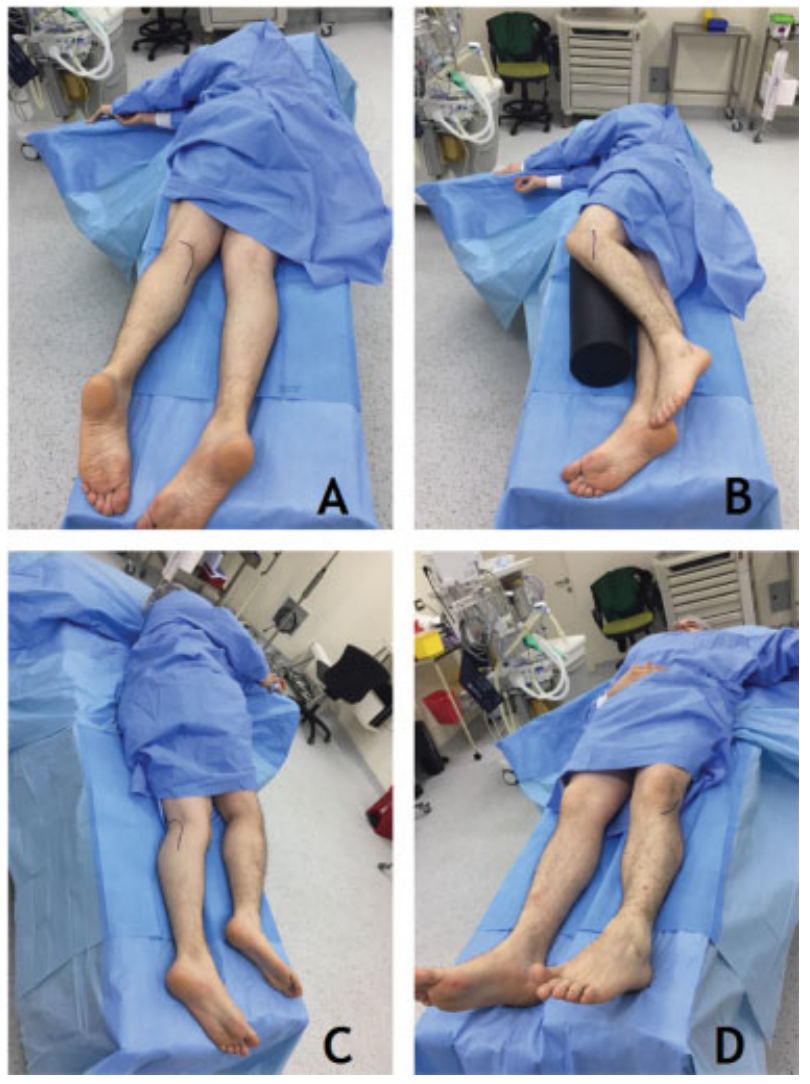

Fig. 4 Posición Flotante: Se preparan ambas extremidades inferiores en el campo quirúrgico con el fin de realizar los cambios de posición durante la cirugía. Se comienza con el abordaje posteromedial. Imágenes A y B. La opción descrita por Luo y cols, ${ }^{6}$ consiste en posicionar al paciente en decúbito semilateral con la extremidad lesionada hacia arriba. Ésta y la pelvis son primero rotadas a una posición en prono para realizar el abordaje posteromedial (A). Luego, la extremidad vuelve a una posición lateral para realizar el abordaje anterolateral (B). Imágenes C y D. Otra alternativa descrita por Chang y cols, ${ }^{62}$ posiciona la extremidad lesionada hacia abajo. Para ello, la cadera contralateral es flectada y rotada hacia el prono por sobre la extremidad lesionada, la que rota lateralmente con esas maniobras, permitiendo el acceso a la región posterior de la rodilla (C). Tras el abordaje posteromedial, se retorna al paciente a la posición supino para el abordaje anterolateral (D).

Otros autores ${ }^{59,64}$ recomiendan que tras manejar la columna posterior en prono, se debe reposicionar al paciente en decúbito supino con una nueva preparación del campo quirúrgico para proseguir con el manejo inmediato o diferido de la columna anterolateral, principalmente por ser más adecuado para la fisiología de un paciente con comorbilidades crónicas (patología pulmonar, obesos), o con lesiones sistémicas agudas asociadas y su monitorización intraoperatoria; más eficiente para el uso de distractores articulares o adquisición de radiografías intraoperatorias y más predecible para el cirujano, ya que de esa manera, se logra convertir la fractura a un rasgo más simple, que puede manejarse con un abordaje anterolateral tradicional y en posición anatómica, más familiar para la mayoría de los traumatólogos, permitiendo una mejor estimación del alineamiento de la extremidad. La desventaja de esa modalidad es que no existe posibilidad de ajuste de la reducción y fijación posteromedial una vez que el paciente es 
reposicionado en supino. Además, prolonga el tiempo quirúrgico a consecuencia del cambio de posición y segunda preparación del campo operatorio.

El principal objetivo del abordaje anterolateral es elevar la depresión articular conminuta con adición de injerto óseo en los casos de gap residual y fijar la cortical lateral con una placa bloqueada, tornillos largos y subcondrales que protejan la reducción, mantengan unidos ambos platillos y aseguren su contacto con la diáfisis tibial.

\section{Abordajes Posteromediales}

En 1994, Georgiadis ${ }^{8}$ describe el primer abordaje posteromedial para el manejo de una fractura. Luego, De Boeck y Opdecam ${ }^{9}$ realizaron modificaciones al abordaje en la región posterior de la rodilla en forma de "S" descrito por Trickey ${ }^{65}$ en 1968 para el manejo de lesiones del ligamento cruzado posterior, con el fin de acceder de manera segura a ese fragmento. Lobenhoffer y cols ${ }^{10}$ en 1997 describe un abordaje en supino entre los ligamentos colateral medial y oblicuo posterior para el manejo del fragmento posteromedial. Carlson ${ }^{11,66}$ no divide la cabeza medial del gastrocnemio en su abordaje en prono, a diferencia de autores previos ${ }^{8,9}$ y agrega una capsulomotía submeniscal con el fin de visualizar la articulación.

En 2003, Galla y Lobenhoffer ${ }^{12}$ perfeccionaron el abordaje posteromedial directo en prono. Se dio a conocer en la literatura en idioma inglés el año 2007 por Fakler y cols. ${ }^{67}$ Demostraron que es posible una disección segura que evita el paquete neurovascular poplíteo y permite una excelente visualización de la columna posteromedial, otorgando una gruesa cobertura del implante por partes blandas del compartimiento posterior de la pierna. Se realiza una incisión longitudinal de 8 a $10 \mathrm{~cm}$ que parte sobre la línea articular y se extiende a lo largo del borde medial del músculo gastrocnemio medial, el cual es retraído lateralmente y utilizado para proteger las estructuras neurovasculares que avanzan profundo y lateral a su vientre muscular. Se desarrolla un plano intermuscular entre el gastrocnemio medial y el semimembranoso sin necesidad de disecar la pata de ganso por anterior (- Fig. 5A). Finalmente, se realiza una elevación subperióstica del músculo poplíteo de distal a proximal, exponiendo la línea de fractura (-Fig. 5B).
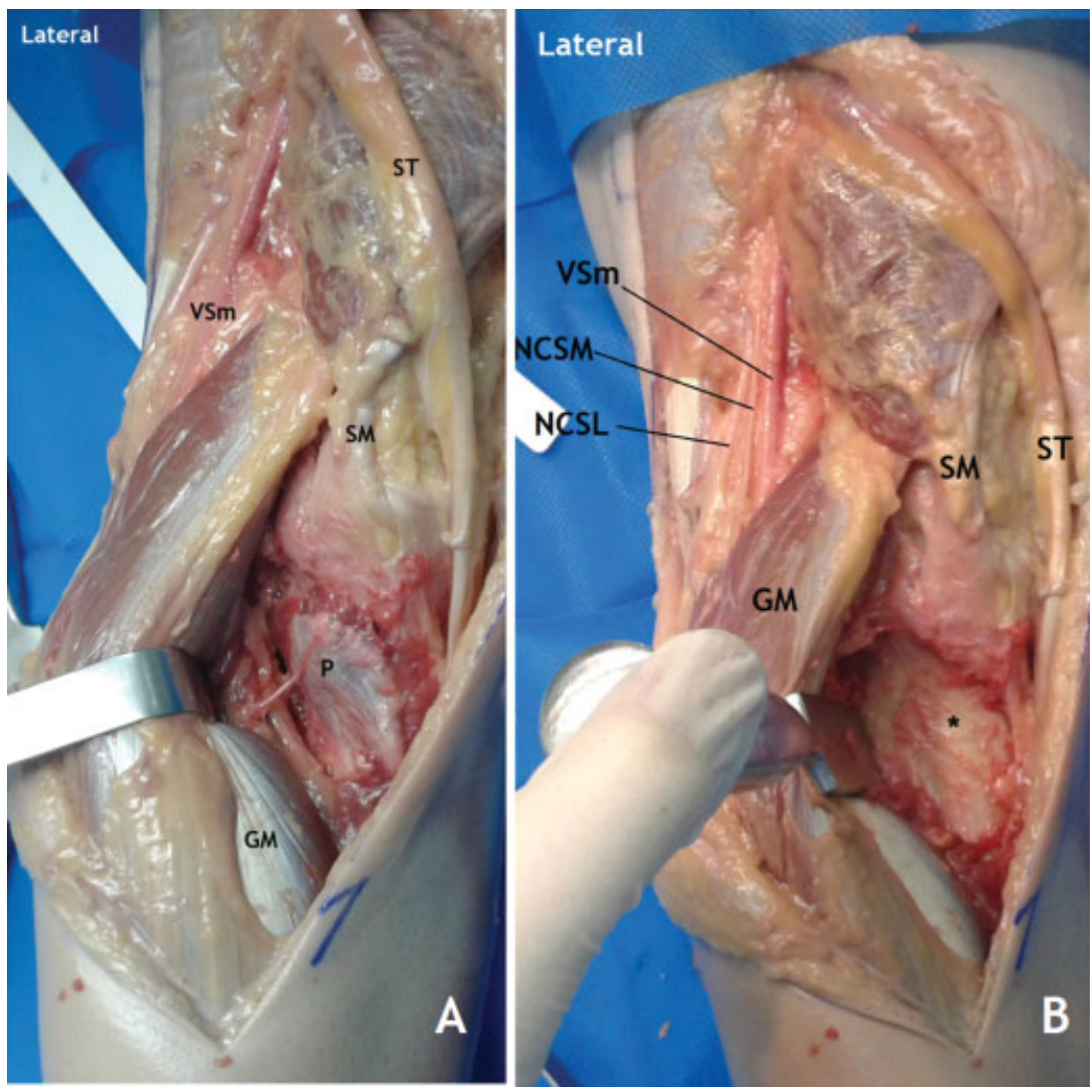

Fig. 5 Abordaje posteromedial en prono. Imagen A. Se realiza la disección hasta la fascia posterior de la pierna, la cual se incide entre el gastrocnemio medial (GM) y la pata de ganso (tendón semitendinoso, ST). Se establece un flap fasciocutaneo de espesor total con el fin de proteger el nervio cutáneo sural medial (rama directa del nervio tibial) y la vena safena menor (VSm), que se encuentra medial a ése. El ligamento colateral medial permanece intacto anterior y profundo a los tendones de la pata de ganso, los cuales se pueden retraer con drenaje de Penrose o cinta vascular. Imagen B. En la disección más profunda, el GM y sóleo son elevados y retraídos posterior y lateralmente con un Hohmann instalado en la cortical posterolateral de la tibia proximal. Debe evitarse una retracción forzada con el fin de no lesionar por elongación las estructuras neurovasculares. El músculo poplíteo (P) y sóleo son elevados a lo largo de su margen medial progresando lateralmente, exponiendo por completo el aspecto posterior de la tibia proximal $(*)$, sin necesidad de realizar una tenotomía del GM. Las estructuras neurovasculares poplíteas están protegidas entre el músculo sóleo y poplíteo. Si se moviliza la cabeza del GM, es importante identificar y proteger su pedículo vascular, a aproximadamente $5 \mathrm{~cm}$ de su inserción en el fémur. SM: semimembranoso, NCSM: nervio cutáneo sural medial (rama directa del nervio tibial), NCSL: nervio cutáneo sural lateral (rama directa del nervio peroneo común). 
Barei y cols ${ }^{60}$ y luego Weil y cols, ${ }^{61}$ describen el abordaje posteromedial en posición supina. Con la extremidad rotada externo, se realiza una incisión a lo largo del margen posteromedial de la tibia, comenzando $3 \mathrm{~cm}$ proximal a la línea articular y extendiéndose distalmente lo necesario para lograr una adecuada reducción. El nervio safeno se encuentra anterior a la vena safena mayor y deben ser protegidos durante la disección, la cual debe atravesar la fascia del sartorio. La línea de fractura comúnmente se encuentra a nivel de la pata de ganso en la porción metafisio diafisiaria de la tibia, en relación directa con el ligamento colateral medial superficial. Los tendones de la pata de ganso pueden ser retraídos o tenotomizados según la preferencia del cirujano. Los músculos gastrocnemio medial y sóleo son retraídos hacia posterior. Eso expone la unión entre la fascia poplítea (posterior y distal), la inserción del semimembranoso (posterior y proximal) y el borde posterior del ligamento colateral medial superficial. Utilizando la disección subperióstica para evitar una lesión de la arteria genicular inferomedial, se eleva la inserción del músculo poplíteo desde el margen posteromedial de la tibia, permitiendo la visualización del foco de fractura. Generalmente, la reducción de la línea articular es realizada de forma indirecta mediante estabilización del ápex de la fractura con apoyo fluoroscópico. Es una opción, realizar una artrotomía submeniscal posterior para evaluar la reducción mediante visualización directa de la línea articular.

\section{Abordajes Posterolaterales}

El fragmento posterolateral se ha percibido como el más difícil de abordar debido a la falta de experiencia en el manejo específico de esa lesión, por su baja incidencia y su ubicación en estrecho contacto con estructuras nobles como el nervio peroneo común, el paquete vascular tibial anterior y la arteria genicular inferolateral. Además, existe un espacio limitado para la visualización y contención de ese fragmento por vías de abordaje clásicos (indirectos). Sin embargo, varios estudios han demostrado que las tasas de complicaciones con los abordajes posterolaterales, no son superiores respecto a los abordajes tradicionales. ${ }^{66,68-71}$

El primer abordaje directo de esas lesiones, fue descrito en 1979 por Gossling y Peterson ${ }^{7}$ mediante desinserción desde la cabeza de la fíbula del tendón del bíceps femoral y ligamento colateral lateral. Si eso no era suficiente para la exposición ósea, realizaban una osteotomía parcial o total de la cabeza de la fíbula, la cual era utilizada como injerto óseo en el tratamiento de la fractura. En 1993, Tscherne y Lobenhoffer $^{72}$ describen un abordaje que expone el fragmento posterolateral mediante osteotomía fibular. Posteriormente, Lobenhoffer y $\operatorname{cols}^{10}$ y Solomon y cols, ${ }^{69}$ perfeccionaron la técnica transfibular en decúbito supino. La principal crítica a ese tipo de abordajes es la desestabilización de la articulación tibiofibular proximal, el riesgo de lesionar al nervio peroneo común por la cercanía de la osteotomía al cuello de la fíbula ( $2 \mathrm{~cm}$ desde el extremo proximal de la cabeza del peroné), riesgo de inestabilidad iatrogénica de la esquina posterolateral y potenciales problemas derivados de la no unión de la osteotomía tras su fijación.
Inicialmente Carlson ${ }^{11,66}$ con una incisión en forma de " $S$ ", luego Tao y cols ${ }^{73}$ con una incisión local en "L" invertida y finalmente Chang y cols ${ }^{74}$ y Frosch y cols ${ }^{68}$ mediante una incisión longitudinal, fueron los primeros autores en describir abordajes posterolaterales directos, sin necesidad de realizar osteotomía fibular, lo cual disminuye la morbilidad del abordaje y permite una adecuada visualización y reducción de la fractura en decúbito prono $^{66,73,74}$ o decúbito lateral. ${ }^{68}$

Se realiza una incisión de $10 \mathrm{~cm}$ que se inicia proximalmente a lo largo del borde medial del tendón bíceps femoral (BF) y desciende distalmente hasta el borde posteromedial de la fíbula. Se realiza disección a través del tejido subcutáneo y fascia poplítea. Se desarrolla un plano intermuscular entre el BF y el gastrocnemio lateral (GL). En la porción más proximal de ese intervalo, se puede identificar el tejido graso que contiene el nervio peroneo común (NPC), medial al BF. En ese nivel, se origina desde el NPC la rama del nervio cutáneo sural lateral (NCSL). El plano de disección es entre el GL (el cual se retrae medialmente junto al NCSL) y el $\mathrm{BF}$, el cual se retrae lateralmente junto al NPC, que perfora la membrana interósea por debajo de la inserción del BF en la fíbula (-Fig. 6). El GL es el factor limitante de la exposición medial. Dependiendo del patrón de fractura, su origen puede ser dejado intacto, parcialmente elevado (desde inferolateral a superomedial) o tenotomizado y luego reparado.

Distalmente, el músculo sóleo se origina en la tibia y fíbula posterolateral. Su elevación muscular a través de disección roma provee la exposición necesaria de la tibia proximal. Ésta debe limitarse hasta $5 \mathrm{~cm}$ bajo la línea articular con el fin de evitar la trifurcación del paquete vascular tibial anterior (que consiste en 1 arteria y 2 venas) el cual cursa distal al borde inferior del músculo poplíteo y viaja lateralmente a través de la membrana interósea hacia el compartimiento anterior de la pierna (-Fig. 6A).

En un estudio cadavérico reciente, Heidari y cols ${ }^{75}$ concluyeron que la arteria tibial anterior atraviesa la membrana interósea a 46,3 $\pm 9 \mathrm{~mm}$ (rango: $27 \mathrm{~mm}$ a $62 \mathrm{~mm}$ ) de la línea articular del platillo tibial lateral y $35,7 \pm 9 \mathrm{~mm}$ distal a la cabeza de la fíbula, por lo que la disección de esa región debe realizarse con extrema precaución. También debe ser cuidadosa la disección del músculo soleo desde el cuello de la fíbula, con el fin de evitar lesionar el NPC.

El músculo poplíteo y su tendón usualmente cruzan la línea articular a nivel de la fractura. Antes de disecarlo subperiósticamente, se debe identificar y ligar la arteria genicular inferolateral, la cual se encuentra posterior o justo distal a él y se dirige horizontalmente hacia la cabeza del peroné, profunda al ligamento colateral fibular y ligamento popliteofibular hacia anterior.

Sun y cols ${ }^{71}$ realizaron una evaluación anatómica de la región posterolateral del platillo tibial. Concluyeron que el largo del nervio peroneo común expuesto por la incisión posterolateral en "L" de $\mathrm{Tao}^{73}$ fue de $56,48 \mathrm{~mm}$ (rango: $50,12 \mathrm{~mm}$ a $62,56 \mathrm{~mm}$ ). La distancia entre la línea articular y el punto en que el nervio peroneo común atraviesa el cuello de la fíbula fue en promedio $42,18 \mathrm{~mm}$ 

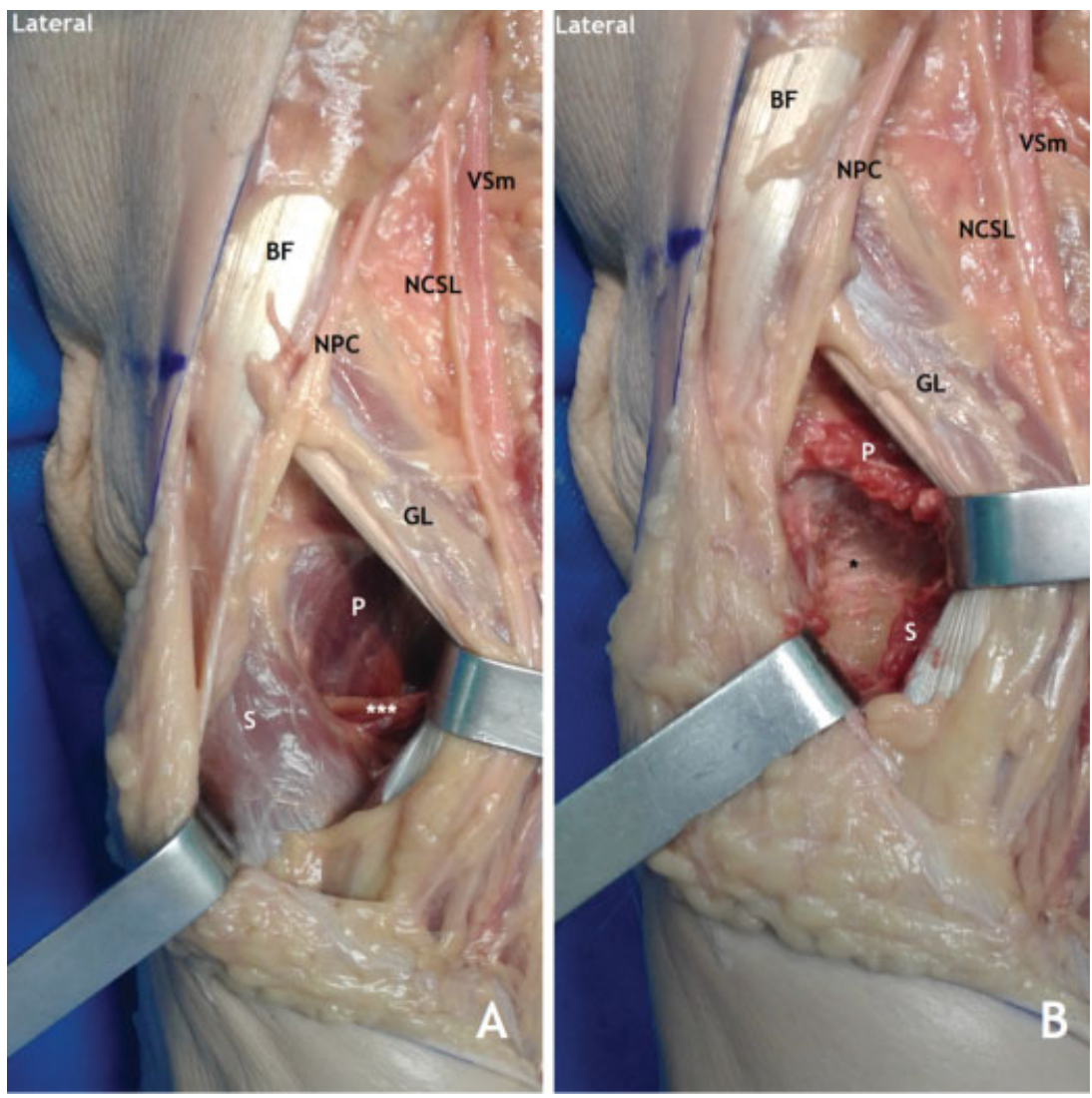

Fig. 6 Abordaje posterolateral directo: Imagen A. La incisión cutánea se realiza a lo largo del borde medial de la cabeza fibular. El tendón del musculo bíceps femoral (BF) se retrae lateralmente para proteger el nervio peroneo común (NPC), que yace en su borde medial. El músculo gastrocnemio lateral $(\mathrm{GL})$ es retraído desde su borde lateral hacia medial. La disección se realiza hacia distal, hasta el punto donde el paquete vascular tibial anterior $\left({ }^{* * *}\right)$ atraviesa la membrana interósea. Se muestran además el nervio cutáneo sural lateral (NCSL), que emerge del nervio peroneo común en el extremo superior de la fosa poplítea, descendiendo para cruzar la superficie de la cabeza lateral del gastrocnemio hacia la línea media de la pierna y la vena safena menor (VSm). Imagen B. El músculo soleo (S) y músculo poplíteo (P) se disecan subperiósticamente y elevan desde la tibia y fíbula proximal. Se retraen el músculo sóleo y poplíteo hacia medial, logrando exponer la tibia posterolateral (*).

(rango: $38,12 \mathrm{~mm}$ a $46,44 \mathrm{~mm}$ ). La arteria genicular inferolateral emergió de la arteria poplítea en promedio a $10,22 \mathrm{~mm}$ (rango: $8,68 \mathrm{~mm}$ a $12,18 \mathrm{~mm}$ ) distal a la línea articular. La distancia promedio entre la superficie articular y la apertura de la membrana interósea para el paquete vascular tibial anterior fue de 48,78 mm (rango: 45,86 a $51,36 \mathrm{~mm}$ ), similar a lo planteado por Heidari y cols. ${ }^{75}$

Si el rasgo de fractura posterolateral ocurre asociado a una fractura que compromete la columna anterolateral, se puede combinar la disección subcutánea de ambos abordajes (posterolateral y anterolateral), mediante una incisión cutánea única a lo largo del cóndilo femoral lateral y la fíbula proximal, como lo describe Frosch y col. ${ }^{68} \mathrm{El}$ control visual de la reducción de la fractura se alcanza utilizando una artrotomía submeniscal lateral estándar. Otra alternativa efectiva para el manejo de esas lesiones es la combinación de un abordaje anterolateral tradicional y un abordaje posteromedial en "L" invertida en posición flotante. ${ }^{76}$ Esa aproximación, entre otras ventajas, evita la formación de un puente cutáneo estrecho entre incisiones independientes (anterolateral y posterolateral), evitando complicaciones de la herida, como la necrosis isquémica de la piel.

\section{Abordaje Posteromedial Directo en L Invertida}

Este abordaje, inicialmente descrito por Burks y Schaffer ${ }^{77}$ en 1990 para el manejo de lesiones del ligamento cruzado posterior, es conocido actualmente como abordaje de Luo, ${ }^{6,63}$ quien lo adaptó en 2010 para el manejo de fracturas de la columna posterior de los platillos tibiales. Su importancia radica en que combina las técnicas descritas previamente a través de un abordaje posteromedial extendido, donde la exposición ósea de la tibia proximal se logra mediante disección subperióstica del músculo poplíteo. Evita la osteotomía fibular y tenotomías de diversas estructuras musculares tales como la pata de ganso y cabeza medial del gastrocnemio, permitiendo una visualización satisfactoria y completa del aspecto posterior de los platillos tibiales, siendo un abordaje seguro y efectivo para tratar un compromiso complejo de la columna posterior que involucre sus fragmentos posteromedial y/o posterolateral (-Figs. 7 y 8). La tasa global de complicaciones con ese abordaje es de un $4,2 \%{ }^{78}$ Además, permite su utilización en combinación con un abordaje anterolateral mediante la posición flotante en 


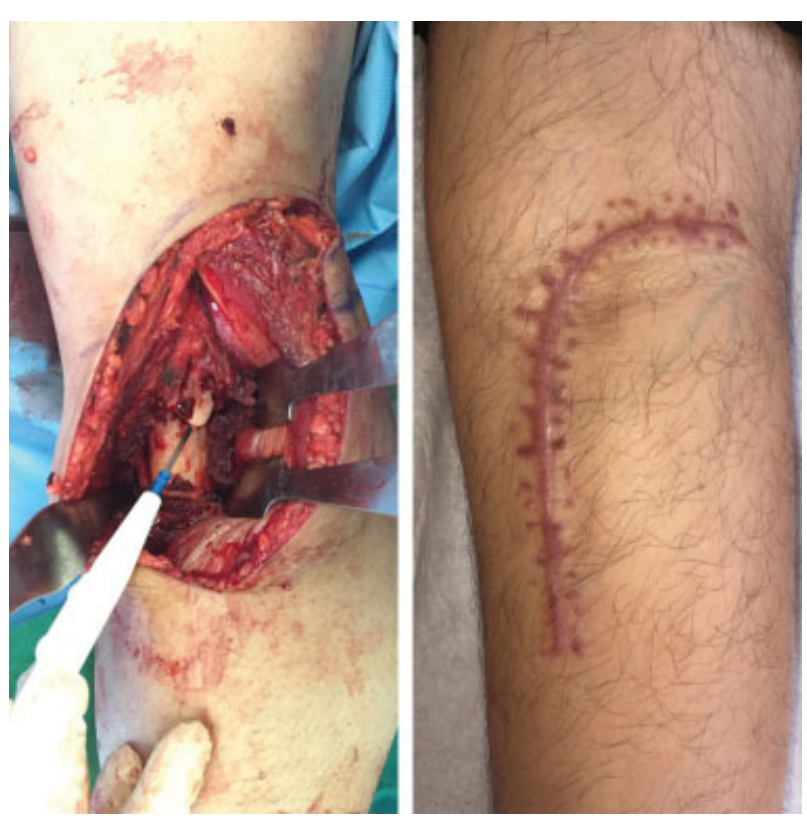

Fig. 7 Abordaje posteromedial en $\mathbf{L}$ invertida: la incisión comienza entre 6 a $8 \mathrm{~cm}$ distal al pliegue de flexión de la rodilla en la región posteromedial de la tibia, a lo largo del borde medial del musculo gastrocnemio medial, continuando proximalmente lateral al tendón semitendinoso. La incisión asciende justo sobre el pliegue de flexión de la rodilla paralelo a las líneas de Langer, extendiéndose lateralmente sobre el centro de la fosa poplítea hasta la línea media. Se deben evitar ángulos agudos en la transición entre la incisión longitudinal y la transversa para evitar necrosis cutánea. Para un fragmento posteromedial, la placa de sostén se instala usualmente en dirección longitudinal, mientras que para un fragmento posterolateral, la placa se instala habitualmente de forma oblicua. Tras la reducción y osteosíntesis, se recomienda dejar abierta la fascia profunda con el fin de evitar un síndrome compartimental y realizar una hemostasia prolija, liberando la isquemia (si se utiliza) previo al cierre cutáneo.

fracturas complejas de platillos tibiales con compromiso de ambas columnas. 6,62,63

En una carta al editor, ${ }^{79}$ el grupo de Chang, quienes fueron uno de los primeros autores en describir el abordaje posterolateral directo sin necesidad de osteotomía de la fibula, ${ }^{74}$ reconocen una alta incidencia de reoperación con su técnica debido a la osteosíntesis sintomática tras la consolidación de la fractura. Reportaron lesión venosa significativa en relación al límite inferior de la disección durante la remoción de la placa y los tornillos (a nivel de la trifurcación), por lo que actualmente recomiendan la exposición del fragmento posterolateral mediante un abordaje de Luo e instalación de una placa de sostén en posición oblicua, de medial a lateral, lo cual está validado biomecanicamente. ${ }^{48}$

En un estudio reciente de Pierrie y cols, ${ }^{80}$ se determinó el área de superficie ósea visible con interposición mínima de tejidos blandos en cada uno de los abordajes más relevantes descritos para acceder al fragmento posterolateral, siendo de $3,87 \pm 2,67 \mathrm{~cm}^{2}$ para el abordaje directo de Frosch, ${ }^{68} 6,87 \pm 1,65 \mathrm{~cm}^{2}$ para el abordaje transfibular de Solomon $^{69}$ y $19,71 \pm 6,34 \mathrm{~cm}^{2}$ para el abordaje de Luo, concluyendo que ese último, permite un acceso amplio a la metáfisis proximal de la tibia, incluyendo los fragmentos posterolaterales.

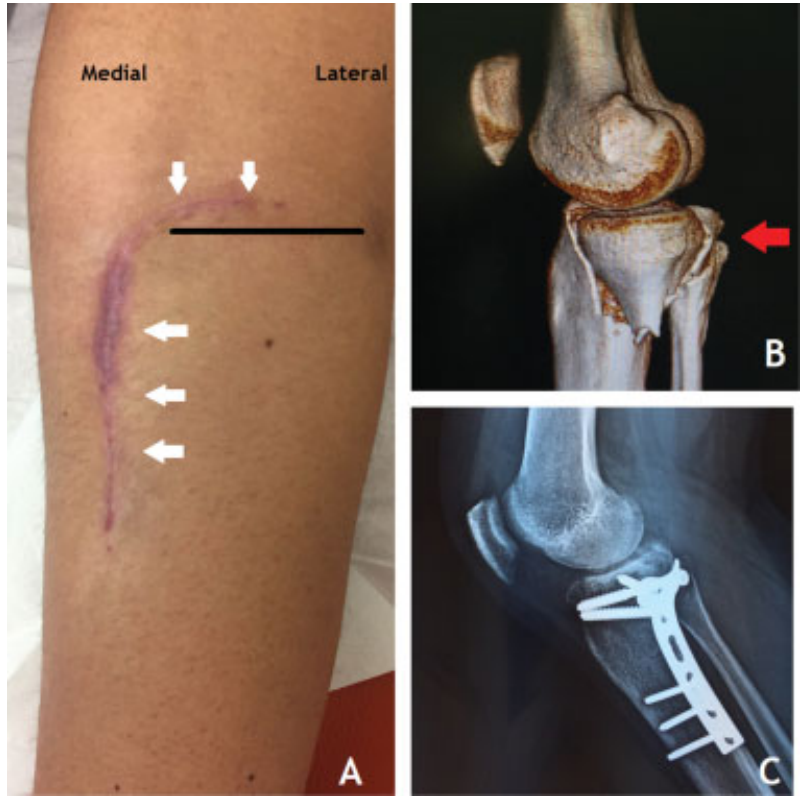

Fig. 8 Abordaje posteromedial en L invertida (continuación). Imagen A. Resultados del abordaje (flechas blancas) en paciente de sexo femenino. Se debe respetar el pliegue de flexión de rodilla (línea negra) para evitar contracturas. Imagen B y C. Este abordaje es de indicación ideal en fracturas con compromiso de columna posteromedial aislada (flecha roja) para reducción y osteosíntesis con placa de sostén.

\section{Conclusiones}

Las fracturas de platillos tibiales con compromiso de la columna posterior son frecuentes $y$ se asocian a mecanismos de alta energía. Se caracterizan por presentar fragmentos altamente inestables y difíciles de reducir mediante abordajes convencionales. La falla en la fijación de esos fragmentos se asocia a la inestabilidad de la rodilla independiente de su tamaño o grado de desplazamiento. Se han desarrollado abordajes específicos para su tratamiento, los que implican un conocimiento acabado de la anatomía de la zona con el fin de evitar lesiones neurovasculares asociadas. Dichos abordajes, no se han asociado a mayores tasas de complicaciones, por lo que su uso se ha hecho habitual en el manejo de la columna posterior, mejorando los resultados clínicos al lograr restablecer de forma más reproducible la biomecánica normal de la rodilla.

\section{Agradecimientos}

Al Dr. Carlos Stierling del Equipo de Rodilla de Clínica Alemana de Santiago, por facilitar las dependencias del Departamento de Anatomía de la Universidad del Desarrollo, para la disección de una pieza cadavérica utilizada en este artículo. Al Equipo de Rodilla de Clínica Alemana de Santiago y Hospital Clínico Mutual de Seguridad por motivar al autor principal a realizar una revisión detallada de la literatura a partir de sus enseñanzas. Al Servicio de Ortopedia y Traumatología del Hospital Ricardo Valenzuela Sáez de Rengo, por permitir la utilización de imágenes intraoperatorias en este artículo. 


\section{Bibliografía}

1 Schatzker J, McBroom R, Bruce D. The tibial plateau fracture. The Toronto experience 1968-1975. Clin Orthop Relat Res 1979; (138):94-104

2 Müller ME, Nazarian S, Koch P, Schatzker J. The Comprehensive Classification of Fractures of Long Bones. Springer-Verlag Berlin Heidelberg; 1990:148-157

3 Charalambous CP, Tryfonidis M, Alvi F, et al. Inter- and intraobserver variation of the Schatzker and AO/OTA classifications of tibial plateau fractures and a proposal of a new classification system. Ann R Coll Surg Engl 2007;89(04):400-404

4 Doornberg JN, Rademakers MV, van den Bekerom MP, et al. Twodimensional and three-dimensional computed tomography for the classification and characterisation of tibial plateau fractures. Injury 2011;42(12):1416-1425

5 Mellema JJ, Doornberg JN, Molenaars RJ, Ring D, Kloen P; Traumaplatform Study Collaborative \& Science of Variation Group. Tibial Plateau Fracture Characteristics: Reliability and Diagnostic Accuracy. J Orthop Trauma 2016;30(05):e144-e151

6 Luo C-F, Sun H, Zhang B, Zeng B-F. Three-column fixation for complex tibial plateau fractures. J Orthop Trauma 2010;24(11):683-692

7 Gossling HR, Peterson CA. A new surgical approach in the treatment of depressed lateral condylar fractures of the tibia. Clin Orthop Relat Res 1979;(140):96-102

8 Georgiadis GM. Combined anterior and posterior approaches for complex tibial plateau fractures. J Bone Joint Surg Br 1994;76(02): 285-289

9 De Boeck H, Opdecam P. Posteromedial tibial plateau fractures. Operative treatment by posterior approach. Clin Orthop Relat Res 1995;(320):125-128

10 Lobenhoffer P, Gerich T, Bertram T, Lattermann C, Pohlemann T, Tscheme $\mathrm{H}$. Spezielle posteromediale und posterolaterale zugänge zur versorgung von tibiakopffrakturen. [Particular Posteromedial and Posterolateral Approaches for the Treatment of Tibial Head Fractures]Unfallchirurg 1997;100(12):957-967

11 Carlson DA. Bicondylar fracture of the posterior aspect of the tibial plateau. A case report and a modified operative approach. J Bone Joint Surg Am 1998;80(07):1049-1052

12 Galla M, Lobenhoffer P. Der direkte dorsale Zugangsweg zur Versorgung posteromedialer Luxationsfrakturen des Tibiakopfes. [The direct, dorsal approach to the treatment of unstable tibial posteromedial fracture-dislocations]Unfallchirurg 2003;106(03): 241-247

13 Yang G, Zhai Q, Zhu Y, Sun H, Putnis S, Luo C. The incidence of posterior tibial plateau fracture: an investigation of 525 fractures by using a CT-based classification system. Arch Orthop Trauma Surg 2013;133(07):929-934

14 Yang G, Zhu Y, Luo C, Putnis S. Morphological characteristics of Schatzker type IV tibial plateau fractures: a computer tomography based study. Int Orthop 2012;36(11):2355-2360

15 Zhai Q, Hu C, Xu Y, Wang D, Luo C. Morphologic study of posterior articular depression in Schatzker IV fractures. Orthopedics 2015; 38(02):e124-e128

16 Zhai Q, Luo C, Zhu Y, et al. Morphological characteristics of splitdepression fractures of the lateral tibial plateau (Schatzker type II): a computer-tomography-based study. Int Orthop 2013;37 (05):911-917

17 Chang SM, Hu SJ, Zhang YQ et al. A surgical protocol for bicondylar four-quadrant tibial plateau fractures. Int Orthop 2014;38(12): 2559-2564

18 Martínez-Rondanelli A, Escobar-González SS, Henao-Alzate A, Martínez-Cano JP. Reliability of a four-column classification for tibial plateau fractures. Int Orthop 2017;41(09):1881-1886

19 Barei DP, O'Mara TJ, Taitsman LA, Dunbar RP, Nork SE. Frequency and fracture morphology of the posteromedial fragment in bicondylar tibial plateau fracture patterns. J Orthop Trauma 2008;22(03):176-182
20 Higgins TF, Kemper D, Klatt J. Incidence and morphology of the posteromedial fragment in bicondylar tibial plateau fractures. J Orthop Trauma 2009;23(01):45-51

21 Zhu Y, Meili S, Dong MJ, et al. Pathoanatomy and incidence of the posterolateral fractures in bicondylar tibial plateau fractures: a clinical computed tomography-based measurement and the associated biomechanical model simulation. Arch Orthop Trauma Surg 2014;134(10):1369-1380

22 Sohn H-S, Yoon Y-C, Cho J-W, Cho W-T, Oh C-W, Oh J-K. Incidence and fracture morphology of posterolateral fragments in lateral and bicondylar tibial plateau fractures. J Orthop Trauma 2015;29 (02):91-97

23 Krause M, Preiss A, Meenen NM, Madert J, Frosch K-H. "Fracturoscopy” is Superior to Fluoroscopy in the Articular Reconstruction of Complex Tibial Plateau Fractures-An Arthroscopy Assisted Fracture Reduction Technique. J Orthop Trauma 2016;30(08):437-444

24 Krause M, Preiss A, Müller G, et al. Intra-articular tibial plateau fracture characteristics according to the "Ten segment classification". Injury 2016;47(11):2551-2557

25 Dhillon MS, Patel S, K P. Simple four column classification can dictate treatment for intra articular tibial plateau fractures much better than ten segment classification. Injury 2017;48(06):1276-1278

26 Krause M, Frosch K-H. Response to the letter-to-the-editor by Dhillon et al. "Simple four column classification can dictate treatment for intra articular tibial plateau fractures much better than ten segment classification", Injury 2017. Injury 2017;48(10):2369-2370

27 Ibrahim DA, Swenson A, Sassoon A, Fernando ND. Classifications In Brief: The Tscherne Classification of Soft Tissue Injury. Clin Orthop Relat Res 2017;475(02):560-564

$28 \mathrm{Kim}$ PH, Leopold SS. In brief: Gustilo-Anderson classification. [corrected]. Clin Orthop Relat Res 2012;470(11):3270-3274

29 DeFrate LE, Sun H, Gill TJ, Rubash HE, Li G. In vivo tibiofemoral contact analysis using 3D MRI-based knee models. J Biomech 2004;37(10):1499-1504

30 Freeman MAR, Pinskerova V. The movement of the normal tibiofemoral joint. J Biomech 2005;38(02):197-208

31 Yildirim G, Walker PS, Sussman-Fort J, Aggarwal G, White B, Klein GR. The contact locations in the knee during high flexion. Knee 2007; 14(05):379-384

32 Immerman I, Bechtel C, Yildirim G, Heller Y, Walker PS, Egol KA. Stability of the posteromedial fragment in a tibial plateau fracture. J Knee Surg 2013;26(02):117-126

33 Cuéllar VG, Martinez D, Immerman I, Oh C, Walker PS, Egol KA. A Biomechanical Study of Posteromedial Tibial Plateau Fracture Stability: Do They All Require Fixation? J Orthop Trauma 2015;29 (07):325-330

$34 \mathrm{Kim} \mathrm{CW}$, Lee CR, An KC, et al. Predictors of reduction loss in tibial plateau fracture surgery: Focusing on posterior coronal fractures. Injury 2016;47(07):1483-1487

35 Wang Y, Luo C, Zhu Y, et al. Updated Three-Column Concept in surgical treatment for tibial plateau fractures - A prospective cohort study of 287 patients. Injury 2016;47(07):1488-1496

36 Honkonen SE. Indications for surgical treatment of tibial condyle fractures. Clin Orthop Relat Res 1994;(302):199-205

37 Moreland JR, Bassett LWHG, Hanker GJ. Radiographic analysis of the axial alignment of the lower extremity. J Bone Joint Surg Am 1987;69(05):745-749

38 Dejour H, Bonnin M. Tibial translation after anterior cruciate ligament rupture. Two radiological tests compared. J Bone Joint Surg Br 1994;76(05):745-749

39 Giannoudis PV, Tzioupis C, Papathanassopoulos A, Obakponovwe $\mathrm{O}$, Roberts C. Articular step-off and risk of post-traumatic osteoarthritis. Evidence today. Injury 2010;41(10):986-995

40 Sun H, Luo CF, Shi HP, et al. Morphological measurements of the posterior surface of the normal proximal tibia in a healthy Chinese population. Knee 2014;21(02):567-572 
41 Anderson CJ, Ziegler CG, Wijdicks CA, Engebretsen L, LaPrade RF. Arthroscopically pertinent anatomy of the anterolateral and posteromedial bundles of the posterior cruciate ligament. J Bone Joint Surg Am 2012;94(21):1936-1945

42 Lin W, Su Y, Lin C, et al. The application of a three-column internal fixation system with anatomical locking plates on comminuted fractures of the tibial plateau. Int Orthop 2016;40(07):1509-1514

43 Berihu BA, Debeb YG. Anatomical variation in bifurcation and trifurcations of sciatic nerve and its clinical implications: in selected university in Ethiopia. BMC Res Notes 2015;8(01):633

44 Kim D, Orron DE, Skillman JJ. Surgical significance of popliteal arterial variants. A unified angiographic classification. Ann Surg 1989;210(06):776-781

45 Jiwanlal A, Jeray KJ. Outcome of Posterior Tibial Plateau Fixation. J Knee Surg 2016;29(01):34-39

46 van den Berg J, Reul M, Nunes Cardozo M, et al. Functional outcome of intra-articular tibial plateau fractures: the impact of posterior column fractures. Int Orthop 2017;41(09):1865-1873

47 Zeng ZM, Luo CF, Putnis S, Zeng BF. Biomechanical analysis of posteromedial tibial plateau split fracture fixation. Knee 2011;18 (01):51-54

48 Zhang W, Luo CF, Putnis S, Sun H, Zeng ZM, Zeng BF. Biomechanical analysis of four different fixations for the posterolateral shearing tibial plateau fracture. Knee 2012;19(02):94-98

49 Eggli S, Hartel MJ, Kohl S, Haupt U, Exadaktylos AK, Röder C. Unstable bicondylar tibial plateau fractures: a clinical investigation. J Orthop Trauma 2008;22(10):673-679

50 Molenaars RJ, Mellema JJ, Doornberg JN, Kloen P. Tibial Plateau Fracture Characteristics: Computed Tomography Mapping of Lateral, Medial, and Bicondylar Fractures. J Bone Joint Surg Am 2015;97(18):1512-1520

51 Ricci WM, Rudzki JR, Borrelli J Jr. Treatment of complex proximal tibia fractures with the less invasive skeletal stabilization system. J Orthop Trauma 2004;18(08):521-527

52 Egol KA, Su E, Tejwani NC, Sims SH, Kummer FJ, Koval KJ. Treatment of complex tibial plateau fractures using the less invasive stabilization system plate: clinical experience and a laboratory comparison with double plating. J Trauma 2004;57 (02):340-346

53 Gosling T, Schandelmaier P, Muller M, Hankemeier S, Wagner M, Krettek C. Single lateral locked screw plating of bicondylar tibial plateau fractures. Clin Orthop Relat Res 2005;439(439):207-214

54 Jiang R, Luo CF, Wang MC, Yang TY, Zeng BF. A comparative study of Less Invasive Stabilization System (LISS) fixation and two-incision double plating for the treatment of bicondylar tibial plateau fractures. Knee 2008;15(02):139-143

55 Ratcliff JR, Werner FW, Green JK, Harley BJ. Medial buttress versus lateral locked plating in a cadaver medial tibial plateau fracture model. J Orthop Trauma 2007;21(07):444-448

56 Yoo BJ, Beingessner DM, Barei DP. Stabilization of the posteromedial fragment in bicondylar tibial plateau fractures: a mechanical comparison of locking and nonlocking single and dual plating methods. J Trauma 2010;69(01):148-155

57 Solomon LB, Stevenson AW, Lee YC, Baird RPV, Howie DW. Posterolateral and anterolateral approaches to unicondylar posterolateral tibial plateau fractures: a comparative study. Injury 2013;44(11):1561-1568

58 Sassoon AA, Torchia ME, Cross WW, Cass JR, Sems SA. Fibular shaft allograft support of posterior joint depression in tibial plateau fractures. J Orthop Trauma 2014;28(07):e169-e175

59 Kottmeier SA, Watson JT, Row E, Jones CB. Staged Fixation of Tibial Plateau Fractures: Strategies for the Posterior Approach. J Knee Surg 2016;29(01):2-11

60 Barei DP, Nork SE, Mills WJ, Henley MB, Benirschke SK. Complications associated with internal fixation of high-energy bicondylar tibial plateau fractures utilizing a two-incision technique. J Orthop Trauma 2004;18(10):649-657

61 Weil YA, Gardner MJ, Boraiah S, Helfet DL, Lorich DG. Posteromedial supine approach for reduction and fixation of medial and bicondylar tibial plateau fractures. J Orthop Trauma 2008;22(05):357-362

62 Chang S-M, Wang X, Zhou J-Q, Huang Y-G, Zhu X-Z. Posterior coronal plating of bicondylar tibial plateau fractures through posteromedial and anterolateral approaches in a healthy floating supine position. Orthopedics 2012;35(07):583-588

$63 \mathrm{He}$ X, Ye P, Hu Y, et al. A posterior inverted L-shaped approach for the treatment of posterior bicondylar tibial plateau fractures. Arch Orthop Trauma Surg 2013;133(01):23-28

64 Tosounidis TH, Giannoudis PV. Letter to the Editor: "A combined posterior reversed L-shaped and anterolateral approach for two column tibial plateau fractures in Caucasians: A technical note". Injury 2016;47(04):983

65 Trickey EL. Rupture of the posterior cruciate ligament of the knee. J Bone Joint Surg Br 1968;50(02):334-341

66 Carlson DA. Posterior bicondylar tibial plateau fractures. J Orthop Trauma 2005;19(02):73-78

67 Fakler JKM, Ryzewicz M, Hartshorn C, Morgan SJ, Stahel PF, Smith WR. Optimizing the management of Moore type I postero-medial split fracture dislocations of the tibial head: description of the Lobenhoffer approach. J Orthop Trauma 2007;21(05):330-336

68 Frosch K-H, Balcarek P, Walde T, Stürmer KM. A new posterolateral approach without fibula osteotomy for the treatment of tibial plateau fractures. J Orthop Trauma 2010;24(08):515-520

69 Solomon LB, Stevenson AW, Baird RPV, Pohl AP. Posterolateral transfibular approach to tibial plateau fractures: technique, results, and rationale. J Orthop Trauma 2010;24(08):505-514

70 Yu B, Han K, Zhan C, Zhang C, Ma H, Su J. Fibular head osteotomy: a new approach for the treatment of lateral or posterolateral tibial plateau fractures. Knee 2010;17(05):313-318

71 Sun H, Luo CF, Yang G, Shi HP, Zeng BF. Anatomical evaluation of the modified posterolateral approach for posterolateral tibial plateau fracture. Eur J Orthop Surg Traumatol 2013;23(07):809-818

72 Tscherne H, Lobenhoffer P. Tibial plateau fractures. Management and expected results. Clin Orthop Relat Res 1993;375(292):87-100

73 Tao J, Hang DH, Wang QG, et al. The posterolateral shearing tibial plateau fracture: treatment and results via a modified posterolateral approach. Knee 2008;15(06):473-479

74 Chang SM, Zheng HP, Li HF, et al. Treatment of isolated posterior coronal fracture of the lateral tibial plateau through posterolateral approach for direct exposure and buttress plate fixation. Arch Orthop Trauma Surg 2009;129(07):955-962

75 Heidari N, Lidder S, Grechenig W, Tesch NP, Weinberg AM. The risk of injury to the anterior tibial artery in the posterolateral approach to the tibia plateau: a cadaver study. J Orthop Trauma 2013;27(04):221-225

76 Sun H, Zhai QL, Xu YF, Wang YK, Luo CF, Zhang CQ. Combined approaches for fixation of Schatzker type II tibial plateau fractures involving the posterolateral column: a prospective observational cohort study. Arch Orthop Trauma Surg 2015;135(02):209-221

77 Burks RT, Schaffer JJ. A simplified approach to the tibial attachment of the posterior cruciate ligament. Clin Orthop Relat Res 1990;(254):216-219

78 Qiu WJ, Zhan Y, Sun H, Xu YF, Wang YK, Luo C. A posterior reversed L-shaped approach for the tibial plateau fractures-A prospective study of complications (95 cases). Injury 2015;46(08):1613-1618

79 Huang YG, Chang SM. The posterolateral approach for plating tibial plateau fractures: problems in secondary hardware removal. Arch Orthop Trauma Surg 2012;132(05):733-734

80 Pierrie SN, Harmer LS, Karunakar MA, et al. Limited Added Value of the Posterolateral Approach. J Knee Surg 2016;29(01):21-27 